\title{
Minireview
}

\section{The bacterial pathogen Listeria monocytogenes: an emerging model in prokaryotic transcriptomics}

\author{
Pascale Cossart ${ }^{\star \ddagger}$ and Cristel Archambaud* ${ }^{* \ddagger}$
}

\author{
Addresses: *Institut Pasteur, Unité des Interactions Bactéries Cellules, Paris F-75015, France. †Inserm U604, Paris F-75015, France. \\ ‡INRA USC2020, Paris F-75015, France. \\ Correspondence: Pascale Cossart: pcossart@pasteur.fr
}

\begin{abstract}
A major challenge in bacterial pathogenesis is understanding the molecular basis of the switch from saprophytism to virulence. Following a recent whole-genome transcriptomic analysis using tiling arrays, an article published in $B M C$ Genomics reports the first use of RNA-seq in Listeria monocytogenes in order to identify genes controlled by sigma $\mathrm{B}$, a transcriptional regulator with a critical role in virulence.
\end{abstract}

See research article http://biomedcentral.com/1471-2164/10/641

A fundamental goal in infection biology is to identify the attributes of a pathogen that allow it to establish an infection and the mechanisms whereby this is achieved. Listeria monocytogenes is a soil bacterium that lives in decaying vegetation and can contaminate food products. In healthy individuals $L$. monocytogenes causes gastroenteritis, but in immunocompromised individuals it can cause meningitis, with a high mortality rate, and in pregnant women it can lead to abortion. It is closely related to Bacillus subtilis, which survives adverse conditions by sporulating, and although $L$. monocytogenes does not sporulate, it can survive and even replicate in harsh environments, including those, such as low $\mathrm{pH}$, low temperature and high salt, that are used to control food contamination. Listeria is ubiquitous in the environment but was discovered only in 1926, as the cause of an epidemic affecting rabbits and guinea pigs in animal-care houses (Figure 1). In the infected host, this bacterium is mostly intracellular, owing to its capacity to resist macrophage killing and to its exquisite property to invade a variety of non-phagocytic cells, including epithelial cells such as the enterocytes of the intestine [1].

Because of its intracellular niche, Listeria cannot be reached by antibodies, and the pioneering studies of Mackaness in 1960 showed that recovery from infection and protection against a secondary infection are mediated by $\mathrm{T}$ lymphocytes, now known to activate bactericidal mechanisms in macrophages and to kill infected cells. Listeria has since been and still is an important tool in the study of T-cell responses. Two decades after Mackaness' discoveries, a combination of molecular biology, cell biology and classical genetic approaches has been used to address the molecular basis of Listeria virulence. Several key factors contributing to cellular invasion, escape from the vacuole, and intra- and intercellular dissemination have been identified and characterized. New concepts in infectious biology rapidly emerged, and the remarkable virulence toolkit revealed by in vitro and in vivo studies has made Listeria a model organism in the emerging discipline of cellular microbiology. In a recent study published in BMC Genomics, Boor and colleagues (Oliver et al. [2]) apply high-throughput 'deep' sequencing to investigate the transcriptome characteristic of the stress response of $L$. monocytogenes, in particular its regulation by the 'alternative' sigma factor B, by comparing a standard strain with an isogenic mutant lacking sigma B.

\section{Genome studies and the study of Listeria biodiversity and virulence}

The first complete genome sequence of $L$. monocytogenes was determined by a European consortium in 2001, at the same time as that of Listeria innocua, a closely related non-pathogenic species [3]. The Listeria genus contains only six species, of which two are pathogenic - L. monocytogenes and Listeria ivanovii, an animal pathogen. The genome of the sequenced strain (EGD-e) of L. monocytogenes is $2,944,528$ bp in size and contains 2,853 proteincoding genes (genes encoding polypeptides larger than 50 amino acids). The initial comparison of $L$. monocytogenes EGD-e and L. innocua sequences revealed strong conservation of gene organization, relatively few insertion elements, and the absence of typical bacterial 'pathogenicity islands', that is, large clusters of virulence genes. Instead, deletions and insertions have led to a general organization of a conserved backbone with multiple interspersed speciesspecific islets.

The sequences of three other clinical strains of L. monocytogenes were subsequently determined by the Institute of Genomic Research in 2004 [4]. Comparison with strain EGD-e revealed that genome organization is highly 


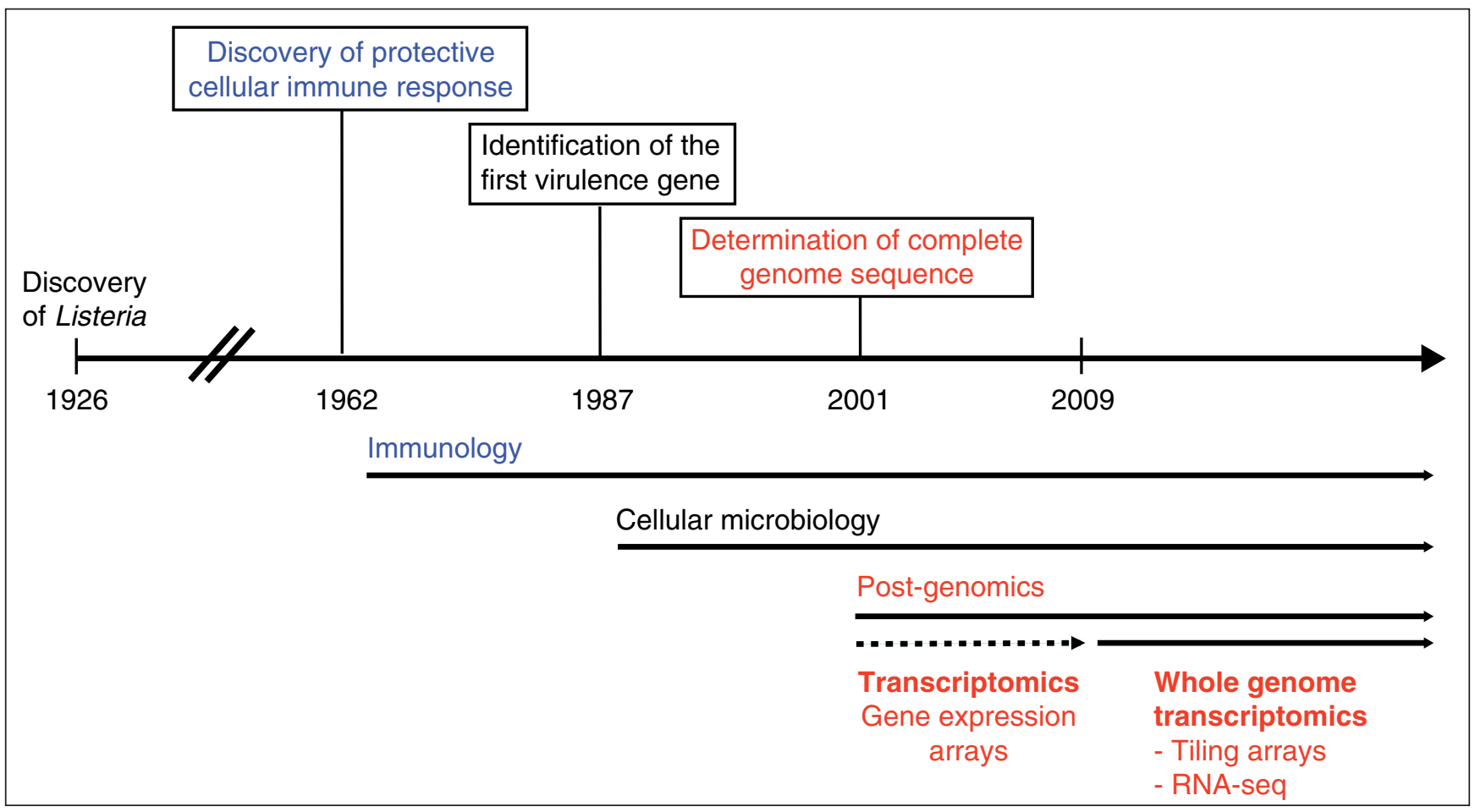

Figure 1

Key dates and events, and related research areas on Listeria since its discovery in 1926.

conserved among strains, with a large number of orthologous genes. However, as initially observed in the comparison between $L$. monocytogenes and $L$. innocua, the genomes also possess a considerable number of strainspecific traits, most of them organized into many small plasticity zones. For example, 2,499 genes are conserved in the four L. monocytogenes genomes, of which 2,394 are also present in L. innocua. Further comparisons are now possible because the Broad Institute has recently accomplished the sequence of $18 \mathrm{~L}$. monocytogenes strains of various origins [5].

Post-genomic studies have confirmed as bona fide L. monocytogenes virulence genes several favorite candidates that are absent from $L$. innocua. The best example is $b s h$, which encodes a bile salt hydrolase absent from all L. innocua strains analyzed and which enables bacterial persistence in the intestinal lumen and in deeper organs such as the liver [6]. Current investigations are focused on a series of factors present in L. monocytogenes and absent in L. innocua, and which have been implicated in virulence by animal studies using strains deleted for these factors, but whose precise role in virulence is elusive [1].

\section{Gene expression arrays identify co-regulated or differentially expressed genes}

The complete genome sequence of $L$. monocytogenes has also enabled exhaustive studies of gene expression. The earliest of these interrogated the genes controlled by the transcriptional regulator PrfA in three different L. monocytogenes strains grown in different growth media [7]. PrfA was already known to activate well-characterized major virulence genes such as hly and actA. Transcriptome analysis indicated that the number of transcription units directly regulated by PrfA is probably lower than previously predicted by bioinformatics searches for putative PrfAbinding sites on the genome.

Most importantly, this study [7] highlighted connections between PrfA induction and the sigma B regulon. Sigma B is one of five sigma factors in Listeria. Sigma factors are subunits of prokaryotic RNA polymerase responsible for the recognition of a conserved DNA sequence in a promoter site. Promoter recognition by the polymerase is determined by the transient association of an appropriate sigma factor with the core polymerase in response to conditions affecting the cell. The number of genes regulated by a single sigma factor - its regulon - can be high. Thus, sigma factors are effective for simultaneously regulating large numbers of genes under different conditions. Sigma B- RpoS in Escherichia coli is an 'alternative' sigma factor that regulates the stress response, including the stationary-phase genes.

Over the past decade, the Listeria sigma B regulon, along with the regulons for other transcription regulatory factors 
- for example, sigma 54 [8], and VirR [9] - have been characterized by whole-genome transcriptional profiling. Microarray analysis of the Listeria sigma B regulon showed that it comprises more than 100 genes, including both virulence genes and stress-response genes, many of them being upregulated upon entry into stationary phase $[10,11]$.

Whole-genome profiling has also been used to study Listeria adaptation to various environmental conditions (cold shock, heat shock, alkaline stress, high hydrostatic pressure, milk, and so on), as well the precise adaptive response to the conditions encountered in the host milieu, in epithelial cells and in macrophages [12,13]. A recent study using bacteria isolated from the spleens of intravenously infected mice has identified genes only expressed in vivo and never in vitro, and thus implicated in adaptation to the host [14].

As the high density membranes used in all these studies contained only probes for protein coding genes, the results, although quite comprehensive, only focused on these genes and did not take into account intergenic regions or regions on the strand opposite to annotated open reading frames. Two recent reports have now examined the whole Listeria genome after bacterial growth in different conditions $[2,15]$.

\section{Tiling arrays and deep sequencing reveal the unexpected complexity of the Listeria transcriptome}

Two powerful techniques are now available for genomewide transcriptome analysis: RNA-seq and genomic tiling arrays [16]. In RNA-seq, a population of cellular RNAs is converted to cDNA and subjected to high-throughput sequencing. The sequences are then mapped to the genome to generate a high-resolution transcriptome map reflecting a particular cellular state. A genomic tiling array is a DNA microarray with a set of overlapping oligonucleotide probes that cover the whole genome or a proportion of the genome at high resolution. Here again, cellular RNA is converted to cDNA and hybridized to the array to assess transcription.

Oliver et al. [2] used deep sequencing - that is, RNA-seq to investigate the sigma B regulon in the $10403 \mathrm{~S}$ L. monocytogenes strain, a strain that, like strain EGD-e, has been extensively used to investigate Listeria virulence. The genome sequence of this strain is not totally determined and a reference 'pseudogenome' was created using the genome of strain EGD-e. The authors show that $83 \%$ of annotated genes are transcribed in stationary phase and identified 96 genes with higher expression levels in the wild type than in the sigma B mutant in stationary phase. Of the 67 non-coding RNA elements (ncRNAs) they report as transcribed in stationary phase, 60 had already been described by other investigators and the other seven are new, of which one is absent in EGD-e, four are in intergenic regions and two are in either a protein-coding region or a 5' untranslated region (UTR).

Interestingly, the three genes found by Oliver et al. [2] to be most highly expressed in stationary phase encode noncoding RNAs: two - tmRNA and 6S RNA - are present in all bacteria, and one - LhrA - is specific to Listeria. This latter RNA is an intriguing small RNA that partially overlaps an open reading frame [17]. It is interesting to note that tmRNA and 6S RNAs are implicated in recovery from the stress induced by entry into stationary phase in E. coli and in the adaptation to this new growth phase, respectively. tmRNA tags incompletely translated proteins for degradation and releases stalled ribosomes, while 6S RNA as shown in E. coli mimics an open promoter to bind and sequester the sigma70-containing polymerase, inhibiting transcription at sigma70 promoters and thus increasing transcription at sigma B regulated promoters.

A genomic-tiling array analysis was recently published by our laboratory [15] investigating the transcriptomes of Listeria grown in vitro in several conditions (including exponential and stationary phase, low $\mathrm{O}_{2}$, two temperatures), in vivo (in the intestine of germ-free animals), and ex vivo in blood. The wild-type Listeria strain EGD-e transcriptomes were compared with those of several isogenic mutants, including a prfA mutant, a sigma $B$ mutant and an $h f q$ mutant. Hfq is an RNA-binding protein known to stabilize RNA-RNA hybrids. It is implicated in Listeria virulence [18]. Our study [15] provided the complete Listeria operon map and uncovered many types of RNAs, including 50 small RNAs, antisense RNAs covering several open reading frames, long overlapping 5 ' and 3' UTRs and riboswitches that can act as terminators for upstream genes. PrfA was found to control transcription of virulence genes in the blood, whereas sigma B mediates activation of virulence genes in the intestine, where it regulates several small RNAs, including $\operatorname{sbr} A$, which had been previously identified in silico as regulated by sigma B [19].

\section{Future directions}

Altogether, the new study by Oliver et al. [2] reinforces the emerging view that bacterial transcriptomes are much more complex than expected, and that very careful analysis must be carried out to avoid misinterpretations in both the tiling array and deep sequencing approach [16]. In addition, this recent work reveals that important strain variations occur. These will help to explain the specific properties of a given strain, or correlate structural features with phenotypes, but they will also make it more difficult to elaborate general principles. It is to be expected that the best studies will combine tiling arrays analysis with RNAseq. Such studies should reveal important new RNAmediated controls on important phenomena such as 
virulence or resistance to stress. In this new area of prokaryotic transcriptomics, once again Listeria appears as a tool of choice to address fundamental questions.

\section{Acknowledgements}

We thank Nina Sesto for helpful discussions. Due to journal policy, we have only sparingly referenced the literature and apologize to those whose work we were unable to specifically mention.

\section{References}

1. Hamon M, Bierne $\mathrm{H}$, Cossart P: Listeria monocytogenes: a multifaceted model. Nat Rev Microbiol 2006, 4:423-434.

2. Oliver HF, Orsi RH, Ponnala L, Keich U, Wang W, Sun $Q$, Cartinhour SW, Filiatrault MJ, Wiedmann M, Boor KJ: Deep RNA sequencing of $L$. monocytogenes reveals overlapping and extensive stationary phase and sigma B-dependent transcriptomes, including multiple highly transcribed noncoding RNAs. BMC Genomics 2009, 10:641.

3. Glaser P, Frangeul L, Buchrieser C, Rusniok C, Amend A, Baquero $F$, Berche $P$, Bloecker $H$, Brandt $P$, Chakraborty $T$, Charbit A, Chetouani $F$, Couvé E, de Daruvar A, Dehoux P, Domann E, Domínguez-Bernal G, Duchaud E, Durant L, Dussurget O, Entian KD, Fsihi H, García-del Portillo F, Garrido P, Gautier L, Goebel W, Gómez-López N, Hain T, Hauf J, Jackson D, et al.: Comparative genomics of Listeria species. Science 2001, 294:849-852.

4. Nelson KE, Fouts DE, Mongodin EF, Ravel J, DeBoy RT, Kolonay JF, Rasko DA, Angiuoli SV, Gill SR, Paulsen IT, Peterson J, White O, Nelson WC, Nierman W, Beanan MJ, Brinkac LM, Daugherty SC, Dodson RJ, Durkin AS, Madupu R, Haft DH, Selengut J, Van Aken S, Khouri H, Fedorova N, Forberger H, Tran B, Kathariou S, Wonderling LD, Uhlich GA, et al:: Whole genome comparisons of serotype $4 b$ and $1 / 2 a$ strains of the food-borne pathogen Listeria monocytogenes reveal new insights into the core genome components of this species. Nucleic Acids Res 2004, 32:2386-2395.

5. Broad Institute [http://www.broadinstitute.org/annotation/ genome/listeria_group/MultiHome.html]

6. Dussurget O, Cabanes D, Dehoux P, Lecuit M, Buchrieser C, Glaser P, Cossart P: Listeria monocytogenes bile salt hydrolase is a PrfA-regulated virulence factor involved in the intestinal and hepatic phases of listeriosis. Mol Microbiol 2002, 45:1095-1106

7. Milohanic E, Glaser P, Coppee JY, Frangeul L, Vega $Y$, Vazquez-Boland JA, Kunst F, Cossart P, Buchrieser C: Transcriptome analysis of Listeria monocytogenes identifies three groups of genes differently regulated by PrfA. Mol Microbiol 2003, 47:1613-1625.

8. Arous S, Buchrieser C, Folio P, Glaser P, Namane A, Hebraud $M$, Hechard Y: Global analysis of gene expression in an rpoN mutant of Listeria monocytogenes. Microbiology 2004, 150:1581-1590.
9. Mandin P, Fsihi H, Dussurget $O$, Vergassola M, Milohanic E, Toledo-Arana A, Lasa I, Johansson J, Cossart P: VirR, a response regulator critical for Listeria monocytogenes virulence. Mol Microbiol 2005, 57:1367-1380.

10. Hain $T$, Hossain $H$, Chatterjee SS, Machata S, Volk U, Wagner S, Brors B, Haas S, Kuenne CT, Billion A, Otten S, Pane-Farre J, Engelmann S, Chakraborty T: Temporal transcriptomic analysis of the Listeria monocytogenes EGD-e sigmaB regulon. BMC Microbiol 2008, 8:20.

11. Raengpradub S, Wiedmann M, Boor KJ: Comparative analysis of the sigma B-dependent stress responses in Listeria monocytogenes and Listeria innocua strains exposed to selected stress conditions. Appl Environ Microbiol 2008, 74: 158-171.

12. Chatterjee SS, Hossain H, Otten S, Kuenne C, Kuchmina $\mathrm{K}$, Machata S, Domann E, Chakraborty T, Hain T: Intracellular gene expression profile of Listeria monocytogenes. Infect Immun 2006, 74:1323-1338.

13. Joseph B, Przybilla K, Stuhler C, Schauer K, Slaghuis J, Fuchs TM, Goebel W: Identification of Listeria monocytogenes genes contributing to intracellular replication by expression profiling and mutant screening. J Bacteriol 2006, 188: 556-568.

14. Camejo A, Buchrieser C, Couve E, Carvalho F, Reis O, Ferreira $\mathrm{P}$, Sousa $\mathrm{S}$, Cossart $\mathrm{P}$, Cabanes $\mathrm{D}$ : In vivo transcriptional profiling of Listeria monocytogenes and mutagenesis identify new virulence factors involved in infection. PLOS Pathog 2009, 5:e1000449.

15. Toledo-Arana A, Dussurget O, Nikitas G, Sesto N, Guet-Revillet $\mathrm{H}$, Balestrino D, Loh E, Gripenland J, Tiensuu T, Vaitkevicius K, Barthelemy M, Vergassola M, Nahori MA, Soubigou G, Régnault B, Coppée JY, Lecuit M, Johansson J, Cossart P: The Listeria transcriptional landscape from saprophytism to virulence. Nature 2009, 459:950-956.

16. Sorek R, Cossart P: Prokaryotic transcriptomics: a new view on regulation, physiology and pathogenicity. Nat Rev Genet 2009, 24 Nov [Epub ahead of print].

17. Christiansen JK, Nielsen JS, Ebersbach T, Valentin-Hansen $P$, Sogaard-Andersen L, Kallipolitis BH: Identification of small Hfq-binding RNAs in Listeria monocytogenes. RNA 2006, 12:1383-1396.

18. Christiansen JK, Larsen $M H$, Ingmer H, Sogaard-Andersen L, Kallipolitis BH: The RNA-binding protein Hfq of Listeria monocytogenes: role in stress tolerance and virulence. $J$ Bacteriol 2004, 186:3355-3362.

19. Nielsen JS, Olsen AS, Bonde M, Valentin-Hansen P, Kallipolitis $\mathrm{BH}$ : Identification of a sigma B-dependent small noncoding RNA in Listeria monocytogenes. J Bacteriol 2008, 190:62646270

Published: 30 December 2009

doi:10.1186/jbiol202

(C) 2009 BioMed Central Ltd 